\title{
Enhanced Atom Mobility on the Surface of a Metastable Film
}

\author{
A. Picone, ${ }^{1, *}$ M. Riva, ${ }^{1}$ G. Fratesi ${ }^{2,3}$ A. Brambilla, ${ }^{1}$ G. Bussetti, ${ }^{1}$ M. Finazzi, ${ }^{1}$ L. Duò, ${ }^{1}$ and F. Ciccacci ${ }^{1}$ \\ ${ }^{1}$ CNISM-Dipartimento di Fisica, Politecnico di Milano, Piazza Leonardo da Vinci 32, 20133 Milano, Italy \\ ${ }^{2}$ ETSF, CNISM, Dipartimento di Scienza dei Materiali, Università di Milano-Bicocca, via Cozzi 55, I-20125 Milano, Italy \\ ${ }^{3}$ Dipartimento di Fisica, Università degli Studi di Milano, via Celoria 16, I-20133 Milano, Italy \\ (Received 20 March 2014; revised manuscript received 11 June 2014; published 25 July 2014)
}

\begin{abstract}
A remarkable enhancement of atomic diffusion is highlighted by scanning tunneling microscopy performed on ultrathin metastable body-centered tetragonal Co films grown on $\mathrm{Fe}(001)$. The films follow a nearly perfect layer-by-layer growth mode with a saturation island density strongly dependent on the layer on which the nucleation occurs, indicating a lowering of the diffusion barrier. Density functional theory calculations reveal that this phenomenon is driven by the increasing capability of the film to accommodate large deformations as the thickness approaches the limit at which a structural transition occurs. These results disclose the possibility of tuning surface diffusion dynamics and controlling cluster nucleation and self-organization.
\end{abstract}

DOI: 10.1103/PhysRevLett.113.046102

PACS numbers: 68.43.Jk, 68.55.J-, 81.15.Aa

Atomic diffusion on solid surfaces is a ubiquitous phenomenon that plays a fundamental role in determining a large variety of physical and chemical processes. An example is represented by the self-assembly of metallic nanosized clusters stabilized on either oxide or metallic substrates, which is exploited to produce nanopatterned materials in fast and parallel bottom-up approaches [1,2]. In this frame, adatom diffusion is of paramount importance, since the kinetic constraints typically determine the final morphology of the self-assembled nanostructures. In the field of heterogeneous catalysis, adatom diffusion is particularly relevant in cases where spill-over effects intervene and the reaction proceeds through different steps mediated by spatially separated active sites [3-5]: an enhanced atomic diffusivity can therefore boost the rate of the chemical reactions. On the other hand, in oxide supported metal clusters, catalysis, sintering due to Ostwald ripening, induced by atoms detaching from smaller clusters and diffusing to larger ones, often leads to catalyst deactivation [6].

Various factors have been recognized to influence the diffusivity of atomic species on solid surfaces, for instance, (i) isotropic two-dimensional strain in heteroepitaxial systems, with an in-plane lattice mismatch between the film and the substrate $[7,8]$; (ii) mesoscopic strain, where the relaxations induced by the already nucleated islands significantly affect atomic motion [9]; (iii) availability of small amounts of foreign atoms (surfactants) that, being adsorbed on the substrate prior to the film deposition, can affect the intra- and/or interlayer mass transport [10-13]; (iv) presence of molecular species (chemisorbed or in the gas phase), such as, for instance, water [3] or CO [14]; and (v) long-range adatom-adatom interactions [15-17]. More recently, the enhancement of surface diffusion with acoustic standing waves has been proposed as a method for surface nanostructuring by driving atomic motion [18].
In this Letter, we report on a remarkable increase of atomic diffusion with the thickness of an ultrathin metastable film, namely, tetragonally distorted body-centered (bct) Co grown on $\mathrm{Fe}(001)$. Previous quantitative low energy electron diffraction analysis indicates that, despite the fact that bulk cobalt is characterized by hexagonal close-packed (below $695 \mathrm{~K}$, at $1 \mathrm{~atm}$ ) or face-centered-cubic (above $695 \mathrm{~K}$, at $1 \mathrm{~atm}$ ) crystal structure, metastable bct cobalt thin films can be epitaxially stabilized (up to a thickness of about 10 atomic layers) on top of the Fe(001) surface [19]. These films are characterized by an in-plane lattice constant equal to $a=2.87 \AA$, in registry with the $\mathrm{Fe}(001)$ substrate, and by an out-of-plane parameter $c=2.792 \AA[19,20]$.

From scanning tunneling microscopy (STM) measurements, we show that Co grows on $\mathrm{Fe}(001)$ in a nearly perfect layer-by-layer mode up to nine atomic layers. We further notice a remarkable layer-dependent saturation island density, defined as the maximum number of islands per unit area during nucleation, growth, and the coalescence process. These data suggest that adatom mobility depends on the thickness of the Co film over which the diffusion takes place. Density functional theory (DFT) calculations indeed reveal that the diffusion barrier height progressively drops to 0 . Such a reduction is driven by the possibility of accommodating larger elastic deformations in the ultrathin Co film, which becomes softer and softer as its thickness increases.

The $\mathrm{Fe}(001)$ substrates were obtained by deposition of a thick Fe film $(400 \mathrm{~nm})$ on a $\mathrm{MgO}(001)$ substrate, as described elsewhere [21]. Co films were grown onto these $\mathrm{Fe}(001)$ substrates kept at room temperature by sublimation from pure rods $(4 N)$ under ultrahigh vacuum conditions (pressure: $2 \times 10^{-10} \mathrm{mbar}$ ), at a typical growth rate of about 0.12 equivalent monolayers (MLs) [22] per minute, as monitored by a calibrated quartz microbalance. A wedged 
sample, with the Co thickness varying from 0 to $10 \mathrm{ML}$, was obtained by shadowing the substrate with a movable shutter that was retracted at a constant rate during the growth. The STM measurements were acquired at room temperature and a pressure lower than $1 \times 10^{-10}$ mbar in constant current mode with electrochemically etched tungsten tips. After each acquisition of a STM image, the scan area was changed by means of the coarse motion of the piezoelectric drive, following the wedge from highest to lowest coverage, until the pristine $\mathrm{Fe}$ substrate was reached. This strategy allowed us to correlate with high precision the layer completion measured from the STM images with the absolute thickness of the Co film.

DFT simulations were performed with ultrasoft pseudopotentials and plane waves, as implemented in the QUANTUMESPRESSO package [23], with the Perdew-Burke-Ernzerhof generalized gradient approximation [24] to the exchange and correlation functional. Pseudomorphic Co films were placed on top of a four-layer $\mathrm{Fe}(001)$ slab, optimizing all atomic coordinates except the ones of the bottom two Fe layers. In describing Co adatoms, $(3 \times 3)$ surface supercells were adopted with a $5 \times 5$ sampling of the surface Brillouin zone. In the direction orthogonal to the slab, the system is separated from its replicas by $11 \AA$ thick vacuum portions.

Figure 1(a) displays the sample morphology for a Co coverage of 0.25 ML. Randomly distributed Co islands are visible, with a height of roughly $140 \mathrm{pm}$ indicating the nucleation of single-layer-thick islands. As the film grows, the islands eventually merge and the cobalt film completely wets the Fe substrate (not shown). The same scenario occurs for the nucleation [see Fig. 1(b)] and completion of the second Co layer. In the coverage range investigated in this study, the growth proceeds in a nearly perfect layer-by-layer mode, as shown in Fig. 1(c), which reports the layer filling of the first ten layers as a function of the total coverage.

Information on the Co adatom diffusivity can be extracted by the analysis of the Co island density, which strongly depends on the number of completed layers, as shown in Figs. 2(a)-2(d). We recall that, during the film growth, the nucleation of new islands and the accretion of already nucleated ones are competing phenomena. The first process dominates at low island densities, when a diffusing adatom has a lower probability of binding to an existing island than encountering a second adatom to nucleate a new island. When the island density reaches a critical value (the saturation value), the nucleation process stops and the film growth proceeds exclusively by increasing the lateral size of already existing islands. Further material deposition causes a decreasing of the island density, due to the film percolation, until layer completion occurs and all islands merge in a continuous film. An oscillatory behavior of island density as a function of the film thickness is therefore observed [see Fig. 2(e)], with the saturation island density (hollow red dots) depending on the adatom mobility. Especially interesting here is to notice that such a value
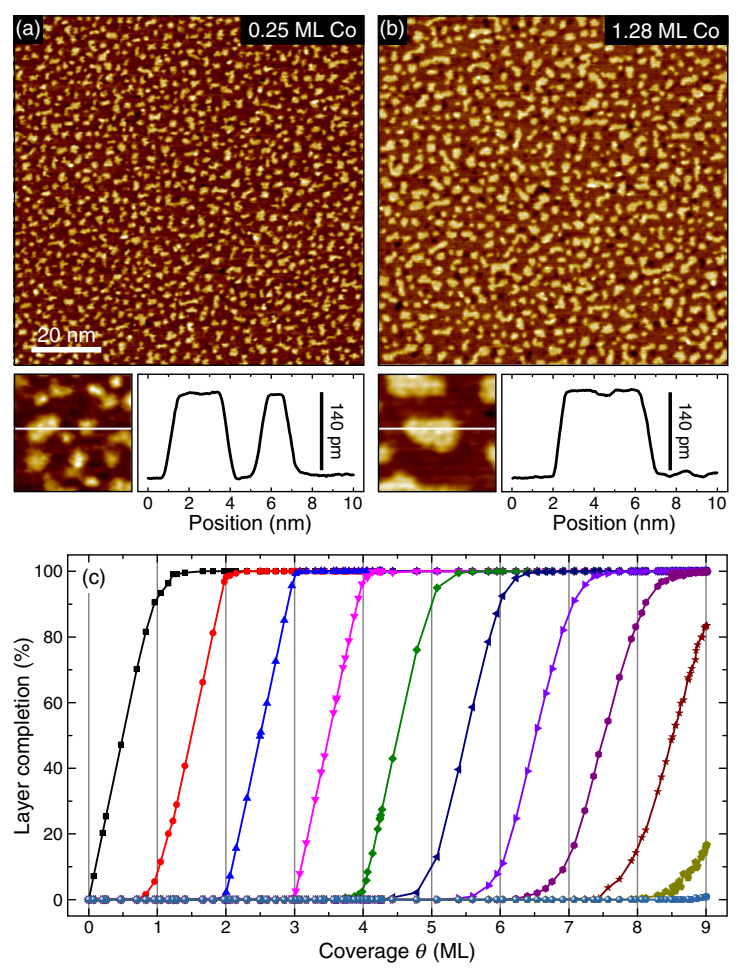

FIG. 1 (color online). (a) Co islands on $\mathrm{Fe}(001)$ for a nominal Co thickness equal to $0.25 \mathrm{ML}$. (b) Morphology of the second Co layer (thickness: $1.28 \mathrm{ML}$ ) on $\mathrm{Fe}(001)$. The insets show that the islands are $1 \mathrm{ML}$ thick. (c) Percentage of layer completion as a function of the total coverage $\theta$, as measured from grain analysis of the STM images. Each symbol and color is associated with a different layer. All samples have been grown at room temperature. STM measurements have been performed in constant current mode $(I=1 \mathrm{nA}, V=1 \mathrm{~V})$.

decreases with coverage by 2 orders of magnitude, starting from $1.17 \times 10^{-1} \mathrm{~nm}^{-2}$ for the nucleated cobalt islands on the bare $\mathrm{Fe}(001)$ substrate, down to $2 \times 10^{-3} \mathrm{~nm}^{-2}$ for eight-layer islands (corresponding to Co adatom diffusion on the seventh layer). Indeed, as it can be seen in Figs. 2(a)-2(d), larger and less abundant islands can be observed as the coverage increases.

More quantitatively, a well-established island-nucleation theory [25-28] relates the saturation island density $n_{s}$ with the diffusion coefficient $D$ through the formula

$$
n_{s}=\eta\left(\frac{\Phi}{D}\right)^{i /(i+2)} e^{E_{i} /(i+2) k_{B} T},
$$

where $\eta$ is a constant parameter, $\Phi$ is the flux rate, $T$ is the substrate temperature during Co deposition, and $i$ is the critical number of atoms above which islands are stable [29]. $E_{i}$ is the formation energy of such a critical-size cluster. In order to get information about the critical nucleus size, we have investigated the effect of a variation in the deposition flux on the saturation island density. In addition, we performed an analysis of the island size distribution at 

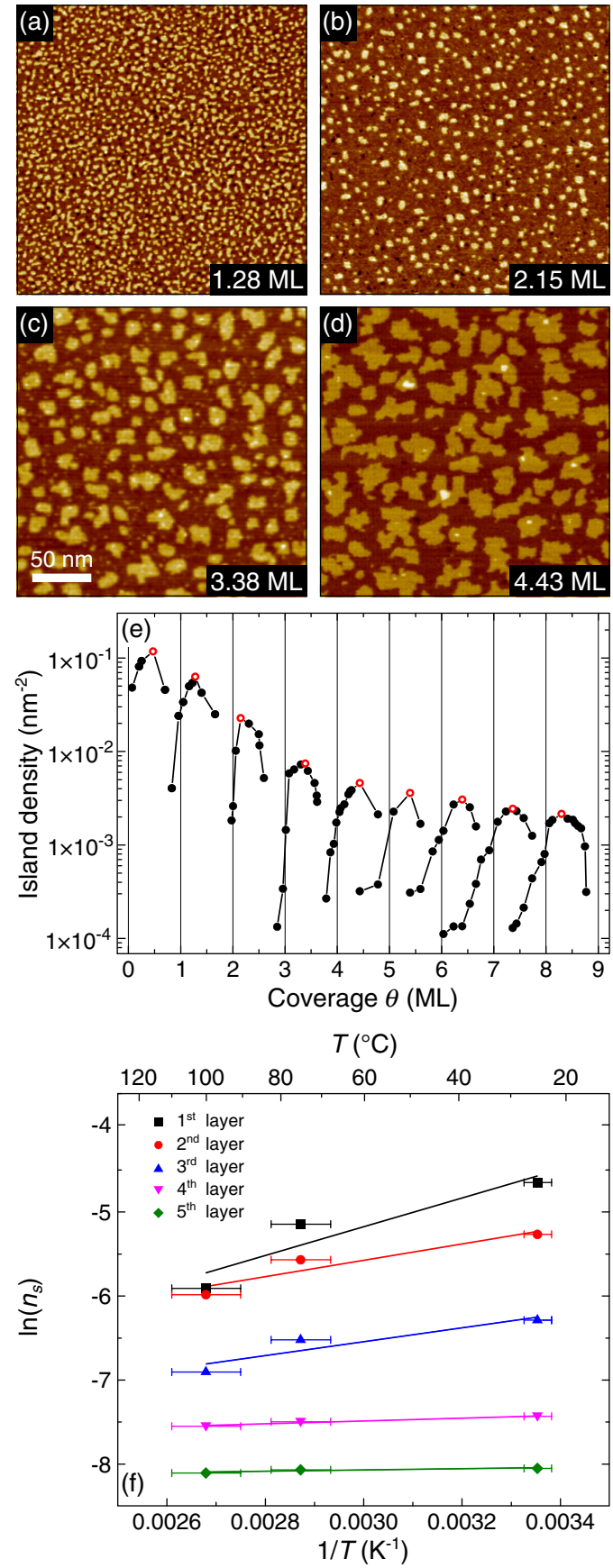

FIG. 2 (color online). STM images collected at coverages for which the Co island saturation density is reached on the (a) first, (b) second, (c) third, and (d) fourth Co layers. The scale [indicated by the white bar in (c)] is the same for all panels. (e) Co island density as a function of the Co coverage $\theta$. The local maxima (hollow red dots) correspond to the saturation island density $n_{s}$ for a given layer. The previous results have been collected on Co films grown at room temperature. (f) Arrhenius plot of saturation island densities (expressed in units of islands per unit lattice cell) derived from STM measurements for islands belonging to the first, second, third, fourth, and fifth layers. The vertical error bars associated with a $\pm 5 \%$ uncertainty in the determination of $n_{s}$ are of the same size of the dots. The lines are linear fits to the data points. saturation [30]. Both these analyses suggest that for the system under consideration, the critical nucleus size is $i=1$, independently on the Co thickness. Our experimental data thus show that the observed island density reduction is unambiguously related with an enhanced adatom mobility associated with a diffusion coefficient $D$ that increases with the film thickness.

Atomic diffusion is a thermally activated process, and, therefore, the dependence of $D$ on the temperature $T$ is described by an Arrhenius equation

$$
D=D_{0} e^{-\left(E_{b} / k_{B} T\right)},
$$

$E_{b}$ being the adatom diffusion barrier and $D_{0}$ a prefactor, depending on the frequency at which the adatoms attempt to overcome the barrier. A critical nucleus size $i=1$ implies $E_{i}=0$; thus, Eq. (1) yields

$$
\ln n_{s}=\frac{E_{b}}{3 k_{B} T}+\frac{1}{3} \ln \left(\frac{\Phi}{D_{0}}\right)+\ln \eta .
$$

Figure 2(f) displays the Arrhenius plot of the saturation island density as a function of $1 / T$. The activation barrier for Co diffusion as a function of the coverage can be extrapolated from the slope of the best fitting line. The experimental $E_{b}^{\exp }$ values are listed in Table I, confirming a dramatic decrease of the surface diffusion barrier as the Co film thickens. Note that the activation barrier for $\mathrm{Co}$ on the bare $\mathrm{Fe}(001)$ surface is about $0.4 \mathrm{eV}$, which is consistent with the value $(0.45 \mathrm{eV})$ found by Stroscio et al. for the homoepitaxial growth of $\mathrm{Fe}$ on $\mathrm{Fe}(001)$ [33].

In order to understand the layer-dependent diffusion rate at the atomic length scale, we have applied DFT to estimate the formation energy $F$ for different Co adatom positions on high-symmetry sites of the (001) surface, namely, hollow, bridge, and top [see Fig. 3(a)]. The adatom formation energy $F$ was computed as

$$
F=\frac{E_{\mathrm{Co} / \mathrm{subs}}^{\mathrm{tot}}-E_{\mathrm{subs}}^{\mathrm{tot}}-\mu_{\mathrm{Co}} N_{\mathrm{Co}}}{N_{\mathrm{Co}}},
$$

TABLE I. Experimental values $E_{b}^{\text {exp }}$ for the Co diffusion barrier as a function of the Co film thickness compared to DFT results for the adatom formation energy in the hollow $F_{H}$ and bridge $F_{B}$ sites. The difference $E_{b}^{\text {calc }}=F_{B}-F_{H}$ estimates the hopping diffusion barrier, which was also computed with frozen substrate coordinates $E_{b}^{\mathrm{fix}}$. Values are in $\mathrm{eV}$.

\begin{tabular}{lccccc}
\hline \hline Coverage & $E_{b}^{\exp }$ & $F_{H}$ & $F_{B}$ & $E_{b}^{\text {calc }}$ & $E_{b}^{\text {fix }}$ \\
\hline $0 \mathrm{ML}$ & $0.45 \pm 0.07$ & 0.15 & 1.36 & 1.21 & 1.37 \\
$1 \mathrm{ML}$ & $0.25 \pm 0.06$ & 0.59 & 1.47 & 0.88 & 1.12 \\
$2 \mathrm{ML}$ & $0.20 \pm 0.05$ & 0.48 & 1.33 & 0.85 & 0.97 \\
$3 \mathrm{ML}$ & $0.05 \pm 0.10$ & 0.60 & 1.15 & 0.55 & 0.97 \\
$4 \mathrm{ML}$ & $0.02 \pm 0.10$ & 0.52 & 0.64 & 0.12 & 0.98 \\
$5 \mathrm{ML}$ & & 0.43 & 0.43 & 0.00 & 0.97 \\
\hline \hline
\end{tabular}




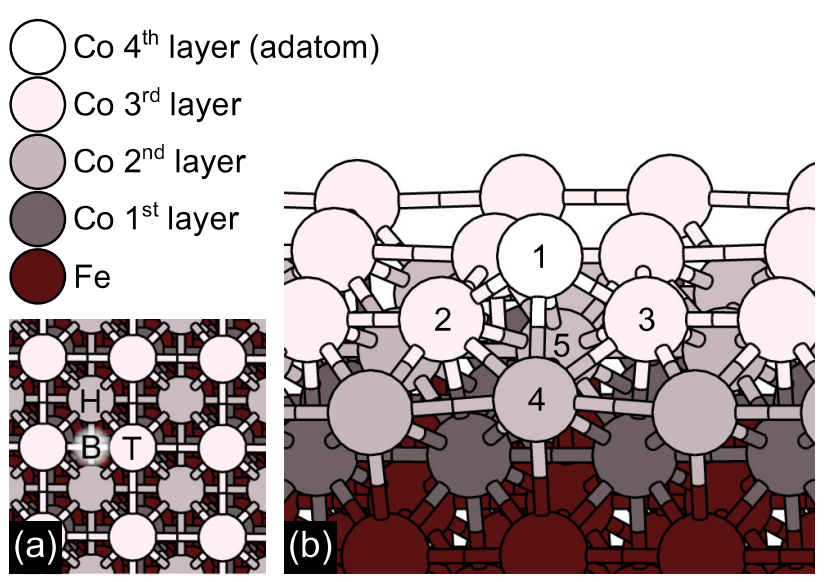

FIG. 3 (color online). Structure of a 3 ML Co film on Fe(001). (a) Top view of the ideal film, with high-symmetry positions indicated: hollow $(H)$, bridge $(B)$, and top $(T)$. (b) Optimized geometry for an adatom (1) occupying the bridge site defined by atoms 2 and 3. Atoms 4 and 5 are the nearest second-layer ones to the adatom.

where $E_{\mathrm{Co} / \text { subs }}^{\text {tot }}$ is the DFT total energy of the combined system, $E_{\text {subs }}^{\text {tot }}$ that of the substrate $(\mathrm{Fe}$ or $\mathrm{Co} / \mathrm{Fe}$ film without adatoms), $\mu_{\mathrm{Co}}$ the chemical potential of $\mathrm{Co}$ adatoms (considering the bulk metal as the reference), and $N_{\text {Co }}$ their number. Lower values indicate more stable configurations. Since the top position is energetically less favorable, the adatom hopping barrier $E_{b}$ is estimated as the difference between the saddle (bridge) energy and the minimum (hollow) one $E_{b}^{\text {calc }}=F_{B}-F_{H}$ (see Table I). It is possible to see that such a hopping barrier, which is maximum on the $\mathrm{Fe}(001)$ substrate $(1.21 \mathrm{eV})$, considerably decreases as the Co film thickness increases and becomes negligible for the $5 \mathrm{ML}$ film. It thus appears that DFT correctly reproduces the order of magnitude and the decreasing trend of the diffusion barriers extrapolated from the experimental data. According to Table I, the lowering of the hopping barrier is mostly due to a remarkable stabilization of the bridge adsorption site, which eventually becomes as stable as the hollow one, with $E_{b}$ varying by about $1 \mathrm{eV}$ in the thickness range from 1 to $5 \mathrm{ML}$.

This thickness dependence can be explained by analyzing the adsorption geometry in the bridge position, which is sketched in Fig. 3(b) for the $3 \mathrm{ML}$ coverage. DFT calculations highlight a significant displacement of the surface Co atoms with respect to their ideal bct(001) position. In particular, the two atoms across the bridge site [labeled 2 and 3 in Fig. 3(b)] are pushed away from each other: their distance is increased from 2.83 to $3.67 \AA$ and they are shifted $0.14 \AA$ closer to the second layer. This allows the adatom (1) to sit closer to the surface, at a distance of only $2.58 \AA$ from the nearest second-layer atoms (4 and 5). The overall displacement induced by the Co adatom can be quantified by the norm of the displacement vector $\|\Delta \mathbf{R}\|=\sqrt{\sum_{j}\left|\Delta R_{j}\right|^{2}}$, which amounts to $0.99 \AA$ and is mostly contributed by atoms in the first two layers. We remark that the use of a larger computation cell would have resulted in even more pronounced effects as a consequence of less constraining boundary conditions, thus partially explaining the mismatch between the experimental and theoretical estimations for $E_{b}$.

The same scenario is qualitatively obtained for all the investigated coverages, with smaller (larger) substrate distortions for thinner (thicker) layers [e.g., $\|\Delta \mathbf{R}\|=$ 0.62(1.46) $\AA$ for 2 (4) ML]. Noteworthily, this deformation of the surface lattice is not associated with a quadratic increase in the elastic energy, as expected from harmonic theory and found, for example, for alkali atoms on metals [34]. On the contrary, the elastic energy that has to be spent to deform the ideal Co film to the optimized coordinates assumed when an adatom is accommodated in a bridge position was found to decrease at higher coverages, indicating a softening of the Co film [30].

A similar analysis was performed for an adatom placed on a hollow adsorption site, showing a smaller deformation and a milder dependence on the film thickness, so that the value of $F_{H}$ is relatively steady. To better identify the role of substrate deformations in the adatom energy, a set of simulations was also run by optimizing the adatom height while keeping the coordinates of the atoms in the film frozen. The result, reported as $E_{b}^{\mathrm{fix}}$ in Table I, shows almost constant values approaching $1 \mathrm{eV}$. This rules out electronic quantum-size effects due to the finite thickness as the possible origin of the coverage-dependent diffusion energy.

The ease in deforming displayed by the Co film is worthy of further considerations. As the film grows, the templating effect of the Fe substrate is less pronounced and elastic energy accumulates to a point where spawning plastic deformations becomes energetically favorable and the film relaxes through a structural transition [35,36]. However, before this critical thickness is reached, the perturbation introduced by inserting an isolated adatom on a bridge site causes the film to rearrange at an incremental energetic cost that becomes smaller and smaller for larger accumulated elastic energy.

In conclusion, we have reported a remarkable layerdependent nucleation behavior on metastable bct Co on $\mathrm{Fe}(001)$. The island density decreases by increasing the film thickness, indicating an enhancement of adatom mobility. DFT calculations show that this is due to a decrease of the energy barrier for adatom hopping and that the observed layer-dependent diffusion barrier and adatom mobility are associated with a mechanical softening of the metastable film approaching the instability limit. In principle, this phenomenon could be a common feature of other metastable structures, as also hinted by the epitaxy of $\mathrm{Mg}$, a hcp metal in the bulk, onto a bcc Mo(001) [37]. The results presented in this Letter might, therefore, disclose the possibility of tuning adatom mobility on substrates with the same chemical identity, by simply varying the film 
thickness. The perspective of tailoring surface diffusion processes would represent an important asset in view of controlling and promoting self-organization processes and heterogeneous catalysis.

This work was partially supported by the Italian Ministry of University and Research through the FIRB Project RBAP115AYN. Computational resources were made available in part by CINECA (Application Codes No. HP10C0TP0R and No. HP10CD51WH).

*andrea.picone@polimi.it

[1] M. Einax, W. Dieterich, and P. Maass, Rev. Mod. Phys. 85, 921 (2013).

[2] H. Brune, Surf. Sci. Rep. 31, 125 (1998).

[3] L. R. Merte, G. Peng, R. Bechstein, F. Rieboldt, C. A. Farberow, L. C. Grabow, W. Kudernatsch, S. Wendt, E. Lgsgaard, M. Mavrikakis, and F. Besenbacher, Science 336, 889 (2012).

[4] W. C. Conner and J. L. Falconer, Chem. Rev. 95, 759 (1995).

[5] G. Kyriakou, M. B. Boucher, A. D. Jewell, E. A. Lewis, T. J. Lawton, A. E. Baber, H. L. Tierney, M. FlytzaniStephanopoulos, and E. C. H. Sykes, Science 335, 1209 (2012).

[6] C. H. Bartholomew, Appl. Catal., A 212, 17 (2001).

[7] H. Brune, K. Bromann, H. Röder, K. Kern, J. Jacobsen, P. Stoltze, K. Jacobsen, and J. Norskov, Phys. Rev. B 52, R14380 (1995).

[8] M. Schroeder and D. Wolf, Surf. Sci. 375, 129 (1997).

[9] D. V. Tsivlin, V. S. Stepanyuk, W. Hergert, and J. Kirshner, Phys. Rev. B 68, 205411 (2003).

[10] A. Picone, A. Brambilla, A. Calloni, L. Duò, M. Finazzi, and F. Ciccacci, Phys. Rev. B 83, 235402 (2011).

[11] A. Calloni, A. Picone, A. Brambilla, M. Finazzi, L. Duò, and F. Ciccacci, Surf. Sci. 605, 2092 (2011).

[12] A. Picone, G. Bussetti, M. Riva, A. Calloni, A. Brambilla, L. Duò, F. Ciccacci, and M. Finazzi, Phys. Rev. B 86, 075465 (2012).

[13] J. Camarero, J. Ferrón, V. Cros, L. Gómez, A. L. Vázquez de Parga, J. M. Gallego, J.E. Prieto, J. J. de Miguel, and R. Miranda, Phys. Rev. Lett. 81, 850 (1998).

[14] G. S. Parkinson, Z. Novotny, G. Argentero, M. Schmid, J. Pavelec, K. Rukan, P. Blaha, and U. Diebold, Nat. Mater. 12, 724 (2013).
[15] K. A. Fichthorn and M. Scheffler, Phys. Rev. Lett. 84, 5371 (2000).

[16] G. Fratesi, G. Alexandrowicz, M. I. Trioni, G. P. Brivio, and W. Allison, Phys. Rev. B 77, 235444 (2008).

[17] S. M. Binz, M. Hupalo, X. Liu, C. Z. Wang, W.-C. Lu, P. A. Thiel, K. M. Ho, E. H. Conrad, and M. C. Tringides, Phys. Rev. Lett. 109, 026103 (2012).

[18] C. Taillan, N. Combe, and J. Morillo, Phys. Rev. Lett. 106, 076102 (2011).

[19] S. K. Kim, C. Petersen, F. Jona, and P. M. Marcus, Phys. Rev. B 54, 2184 (1996).

[20] L. Duò, R. Bertacco, G. Isella, F. Ciccacci, and M. Richter, Phys. Rev. B 61, 15294 (2000).

[21] R. Bertacco, S. De Rossi, and F. Ciccacci, J. Vac. Sci. Technol. A 16, 2277 (1998).

[22] One equivalent monolayer (1 ML) equals the amount of Co atoms required to completely saturate the adsorption sites on the Fe substrate, i.e., about 12.2 atoms $/ \mathrm{nm}^{2}$.

[23] P. Giannozzi et al., J. Phys. Condens. Matter 21, 395502 (2009).

[24] J. P. Perdew, K. Burke, and M. Ernzerhof, Phys. Rev. Lett. 77, 3865 (1996).

[25] J. A. Venables, G. D. T. Spiller, and M. Hanbucken, Rep. Prog. Phys. 47, 399 (1984).

[26] J. G. Amar and F. Family, Phys. Rev. Lett. 74, 2066 (1995).

[27] J. Evans, P. Thiel, and M. Bartelt, Surf. Sci. Rep. 61, 1 (2006).

[28] Islands Mounds and Atoms, edited by T. Michely and J. Krug (Springer, New York, 2004).

[29] The critical size is $i$ if islands with $i+1$ or more atoms are more likely to grow than to dissociate; see Ref. [27].

[30] See Supplemental Material at http://link.aps.org/ supplemental/10.1103/PhysRevLett.113.046102, which includes Refs. [26,31,32], for the determination of the critical cluster size as a function of the coverage and for further details concerning the results of the DFT calculations.

[31] M. Bartelt and J. Evans, Surf. Sci. 298, 421 (1993).

[32] B. Müller, L. Nedelmann, B. Fischer, H. Brune, and K. Kern, Phys. Rev. B 54, 17858 (1996).

[33] J. A. Stroscio, D. T. Pierce, and R. A. Dragoset, Phys. Rev. Lett. 70, 3615 (1993).

[34] G. Fratesi, Phys. Rev. B 80, 045422 (2009).

[35] A. Y. Liu and D. J. Singh, Phys. Rev. B 47, 8515 (1993).

[36] S. Fox and H. J. F. Jansen, Phys. Rev. B 60, 4397 (1999).

[37] M. C. Gallagher, M. S. Fyfield, and S. A. Joyce, Phys. Rev. B 59, 2346 (1999). 


\title{
Enhanced atom mobility on the surface of a metastable film
}

\author{
A. Picone, ${ }^{1}$ M. Riva,${ }^{1}$ G. Fratesi, ${ }^{2,3}$ A. Brambilla,${ }^{1}$ G. Bussetti, ${ }^{1}$ M. Finazzi, ${ }^{1}$ L. Duò, ${ }^{1}$ and F. Ciccacci ${ }^{1}$ \\ ${ }^{1}$ Dipartimento di Fisica and CNISM, Politecnico di Milano, \\ Piazza Leonardo da Vinci 32, 20133 Milano, Italy \\ ${ }^{2}$ ETSF, CNISM, Dipartimento di Scienza dei Materiali, \\ Università di Milano-Bicocca, via Cozzi 55, I-20125 Milano, Italy \\ ${ }^{3}$ Dipartimento di Fisica, Università degli Studi di Milano, via Celoria 16, I-20133 Milano, Italy
}

\section{SUPPLEMENTARY INFORMATION}

\section{Critical-cluster size}

As shown in the inset of Fig. 1(a), the critical-cluster size $i$ is expected to have a characteristic influence on the island size distribution [1]. From Fig. 1(a) it can be seen that the saturation island size distributions are independent of coverage, indicating that the critical cluster size $i$ does not increase with the Co film thickness.

According to Eq. (1), the value of $i$ can be determined by evaluating the saturation island density $n_{\mathrm{s}}$ as a function of the deposition rate $\Phi$ when the the sample is kept at constant temperature. To this aim, we have grown additional samples at room temperature with an evaporation rate $\Phi=\Phi_{1}=0.9 \mathrm{ML} / \mathrm{min}$, corresponding to a sevenfold increase in the flux of Co atoms impinging on the substrate as compered to the rate $\left(\Phi=\Phi_{0}=0.12 \mathrm{ML} / \mathrm{min}\right)$ that was employed to grow the samples discussed in the article. The value of $i$ can then be experimentally determined since, as a consequence of Eq. (1), at constant substrate temperature the ratio $n_{\mathrm{s}}\left(\Phi_{1}\right) / n_{\mathrm{s}}\left(\Phi_{0}\right)$ only depends on $i$ and on $\Phi_{1} / \Phi_{0}$ :

$$
\frac{n_{\mathrm{S}}\left(\Phi_{1}\right)}{n_{\mathrm{S}}\left(\Phi_{0}\right)}=\left(\frac{\Phi_{1}}{\Phi_{0}}\right)^{\frac{i}{i+2}} .
$$

The $n_{\mathrm{s}}\left(\Phi_{1}\right) / n_{\mathrm{s}}\left(\Phi_{0}\right)$ ratio that we obtain by comparing the two sets of samples is displayed in Fig. 1(b) and is in excellent agreement with the value expected for $i=1$ in the whole investigated coverage range. This conclusion is reinforced by reminding that, in the case of a variation of the critical cluster size, on the (001) surface of a cubic substrate one would expect a direct swapping from $i=1$ to $i=3$ due to the transition from single to double-bond breaking [2].

\section{Detail of DFT results}

In Table I we report the computed values, for Co adatoms in the hollow $(\mathrm{H})$ and bridge (B) site, of: (i) the adatom formation energies, $F$ :

$$
F=\frac{E_{\mathrm{Co} / \mathrm{subs}}^{\mathrm{tot}}-E_{\mathrm{subs}}^{\mathrm{tot}}-\mu_{\mathrm{Co}} N_{\mathrm{Co}}}{N_{\mathrm{Co}}}
$$
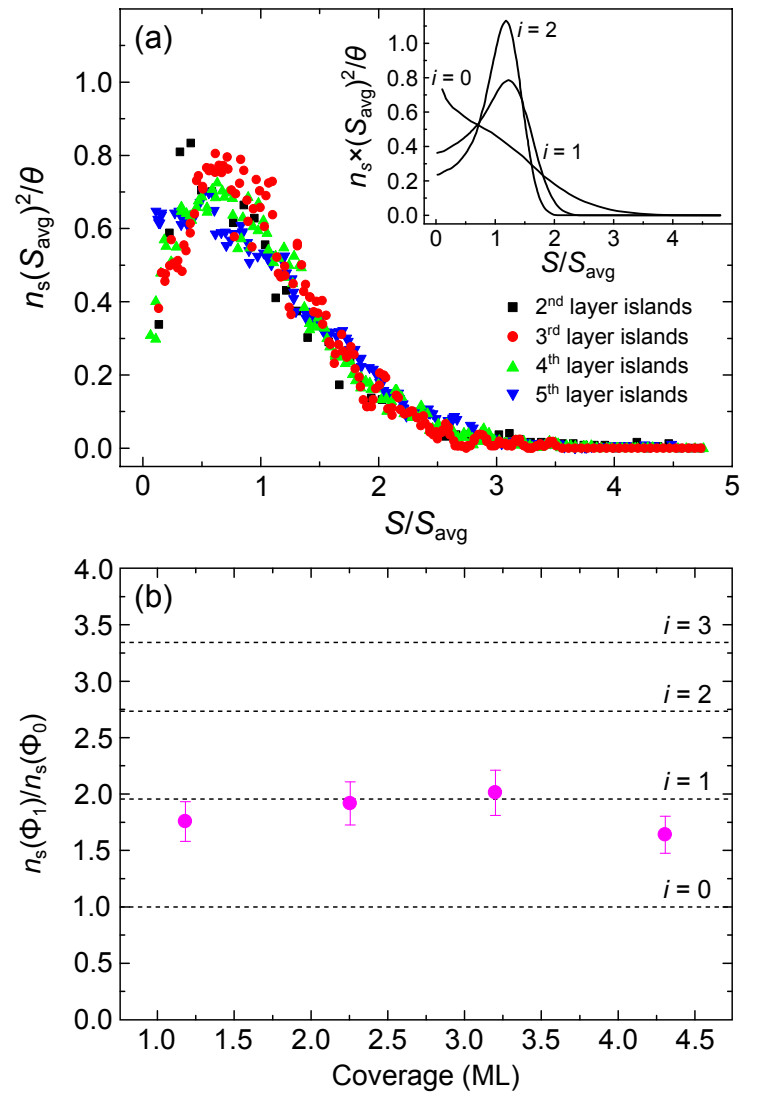

FIG. 1. (a) Scaling analysis of the island size distribution, $n_{\mathrm{s}}$, as a function of the island size $S$ normalized to the average value $S_{\text {avg. The inset shows the corresponding theoretically }}$ expected curves for a critical cluster size $i=0,1,2$, respectively $[3,4]$. (b) $n_{\mathrm{s}}\left(\Phi_{1}\right) / n_{\mathrm{s}}\left(\Phi_{0}\right)$ ratio between the saturation island densities as a function of the Co coverage in two sets of samples grown at room temperature with an evaporation rate $\Phi_{1}=0.9 \mathrm{ML} / \mathrm{min}$ and $\Phi_{0}=0.12 \mathrm{ML} / \mathrm{min}$, respectively. The horizontal dashed lines indicates the values of $\left(\Phi_{1} / \Phi_{0}\right)^{\frac{i}{i+2}}$ for $i=1,2,3$. The error bars are derived from an estimated $\pm 5 \%$ uncertainty associated with the determination of $n_{\mathrm{s}}$.

where $E_{\mathrm{Co} / \mathrm{subs}}^{\text {tot }}$ is the DFT total energy of the combined system, $E_{\text {subs }}^{\text {tot }}$ that of the substrate $(\mathrm{Fe}$ or $\mathrm{Co} / \mathrm{Fe}$ film without adatoms), $\mu_{\mathrm{Co}}$ the chemical potential of $\mathrm{Co}$ adatoms (we take here as a reference the bulk metal) and $N_{\text {Co }}$ their number. Lower values indicate more stable configurations; (ii) the norm of the vector quantifying the displacement of the substrate induced by the Co 


\begin{tabular}{lcccccccccc}
\hline \hline & \multicolumn{3}{c}{ Hollow site } & \multicolumn{3}{c}{ Bridge site } & \multicolumn{3}{c}{ Diffusion barrier } \\
coverage & $F$ & $\|\Delta \mathbf{R}\|$ & $E_{\text {displ }}$ & $F^{\text {fix }}$ & $F$ & $\|\Delta \mathbf{R}\|$ & $E_{\text {displ }}$ & $F^{\text {fix }}$ & $E_{\mathrm{b}}$ & $E_{\mathrm{b}}^{\text {fix }}$ \\
\hline 0 ML & 0.15 & 0.11 & 0.03 & 0.19 & 1.36 & 0.58 & 0.37 & 1.57 & 1.21 \\
$1 \mathrm{ML}$ & 0.59 & 0.10 & 0.04 & 0.64 & 1.47 & 0.58 & 0.38 & 1.76 & 0.88 \\
$2 \mathrm{ML}$ & 0.48 & 0.28 & 0.13 & 0.58 & 1.33 & 0.62 & 0.34 & 1.55 & 0.85 \\
$3 \mathrm{ML}$ & 0.60 & 0.29 & 0.11 & 0.72 & 1.15 & 0.99 & 0.43 & 1.69 & 0.55 & 0.97 \\
$4 \mathrm{ML}$ & 0.52 & 0.48 & 0.11 & 0.68 & 0.64 & 1.46 & 0.24 & 1.65 & 0.12 \\
$5 \mathrm{ML}$ & 0.43 & 0.96 & -0.03 & 0.70 & 0.43 & 1.61 & 0.08 & 1.67 & 0.00 \\
\hline \hline
\end{tabular}

TABLE I. DFT results for the adatom formation energies $F$, the adatom-induced displacement of the substrate atoms $\|\Delta \mathbf{R}\|$, the energy cost of such displacement $E_{\text {displ }}$, the adatom formation energy with frozen substrate coordinates $F^{\mathrm{fix}}$. The diffusion barrier is given by $E_{\mathrm{b}}=F_{\mathrm{B}}-F_{\mathrm{H}}\left(E_{\mathrm{b}}^{\mathrm{fix}}=F_{\mathrm{B}}^{\mathrm{fix}}-F_{\mathrm{H}}^{\mathrm{fix}}\right.$ with frozen substrate coordinates $)$. Values in eV.

adatom, $\|\Delta \mathbf{R}\|=\sqrt{\sum_{I}\left|\Delta R_{I}\right|^{2}} ;$ (iii) the elastic energy cost for such displacement, $E_{\text {displ }}=\bar{E}_{\text {subs }}^{\text {tot }}-E_{\text {subs }}^{\text {tot }}$, where $\bar{E}$ was computed for the substrate in the configuration relaxed in presence of the adatom; (iv) the adatom formation energy obtained with frozen substrate coordinates, $F^{\text {fix }}$ ( (v) the adatom hopping barrier $E_{\mathrm{b}}=F_{\mathrm{B}}-F_{\mathrm{H}}$; (vi) the adatom hopping barrier on a frozen substrate $E_{\mathrm{b}}^{\mathrm{fix}}=F_{\mathrm{B}}^{\mathrm{fix}}-F_{\mathrm{H}}^{\mathrm{fix}}$.

It can be seen that a dramatic increase in substrate deformation at higher coverages does not result in a corresponding increase of the elastic energy (a quadratic dependence on $\|\Delta \mathbf{R}\|$ would be expected from harmonic theory). Therefore, thicker films can accommodate larger deformations, stabilizing the adatom especially in the bridge site and reducing the diffusion barrier. Conversely, by freezing the substrate coordinates, such stabilization is absent and both $F^{\text {fix }}$ and $E_{\mathrm{b}}^{\mathrm{fix}}$ are almost steady, with the obvious exception of the 0 ML case which corresponds to Co diffusion directly on $\mathrm{Fe}(001)$.

[1] M. Bartelt and J. Evans, Surf. Sci. 298, 421 (1993).

[2] B. Müller, L. Nedelmann, B. Fischer, H. Brune, and K. Kern, Phys. Rev. B 54, 17858 (1996).

[3] J. G. Amar and F. Family, Phys. Rev. Lett. 74, 2066 (1995).

[4] J. Evans, P. Thiel, and M. Bartelt, Surf. Sci. Rep. 61, 1 (2006). 